\title{
Article \\ Aminopropylimidazole as an Advantageous Coating in the Synthesis of Functionalized Magnetite Nanoparticles
}

\author{
Alexandrina Nan ${ }^{1, *(\mathbb{D}}$, Iolanda-Veronica Ganea ${ }^{1,2} \mathbb{D}$, Sergiu Macavei ${ }^{1}$ and Rodica Turcu ${ }^{1, *(\mathbb{D})}$ \\ 1 National Institute for Research and Development of Isotopic and Molecular Technologies, \\ 400293 Cluj-Napoca, Romania; iolanda.ganea@itim-cj.ro (I.-V.G.); sergiu.macavei@itim-cj.ro (S.M.) \\ 2 Faculty of Environmental Science and Engineering, "Babeș-Bolyai" University, 400294 Cluj-Napoca, Romania \\ * Correspondence: alexandrina.nan@itim-cj.ro (A.N.); rodica.turcu@itim-cj.ro (R.T.); Tel.: +40-264-584-037 (R.T.)
}

check for updates

Citation: Nan, A.; Ganea, I.-V.;

Macavei, S.; Turcu, R.

Aminopropylimidazole as an

Advantageous Coating in the

Synthesis of Functionalized

Magnetite Nanoparticles.

Nanomaterials 2021, 11, 3276. https://

doi.org/10.3390/nano11123276

Academic Editor:

Alessandro Lascialfari

Received: 25 October 2021

Accepted: 30 November 2021

Published: 2 December 2021

Publisher's Note: MDPI stays neutral with regard to jurisdictional claims in published maps and institutional affiliations.

Copyright: (c) 2021 by the authors. Licensee MDPI, Basel, Switzerland. This article is an open access article distributed under the terms and conditions of the Creative Commons Attribution (CC BY) license (https:/ / creativecommons.org/licenses/by/ $4.0 /)$.

\begin{abstract}
Implementing new methods to prepare magnetite nanoparticles with a covered or uncovered surface has been, and still is, a significant challenge. In this work, we describe a very clear and effortless way for the preparation of magnetite nanoparticles using two types of bases, namely: 1-(3-aminopropyl)imidazole and sodium hydroxide. Fourier transform infrared spectroscopy (FTIR) served as a tool for the structural investigation of the as-prepared magnetite nanoparticles. The morphology of the samples was investigated using Transmission Electron Microscopy (TEM). Comprehensive high-resolution X-ray photoelectron spectroscopy investigations (XPS) were applied as an effective tool for analyzing the composition of the various types of magnetic nanoparticles. Further polymer linkage was accomplished with poly(benzofuran-co-arylacetic acid) on the aminofunctionalized surface of aminopropylimidazole-containing magnetic nanoparticles. The findings are promising for biomedicine, catalysis, and nanotechnology applications.
\end{abstract}

Keywords: magnetite nanoparticles; sodium hydroxide; 1-(3-aminopropyl)imidazole; iron(II) chloride; $X$-ray photoelectron spectroscopy

\section{Introduction}

An intense interest in scientific research and industrial applications is spent developing inorganic nanoparticles due to their unique properties, such as a large surface area/volume ratio and quantum-size effects. Among these, the nanosized iron oxide is a massively promising nanomaterial already used in a wide array of diverse applications, such as catalysis [1-3], adsorbent material of pollutants [4-6], sensing [7,8], optics [9], drug delivery, and cancer diagnosis and treatment [10-13]. In this case, the implementation significantly depends on the shape and size of the nanoparticles. For most applications, magnetic nanoparticles (MNP) have to be coated with stabilizers to allow further functionalization and enhance colloidal and chemical stability. Although useful for several applications, hydroxide stabilizers only provide limited opportunities for further functionalization. Amongst other more versatile stabilizers, alkylamines and imidazole were found to be well-known ligands for metal ions. For example, polyvinylimidazole was successfully used to prepare coated magnetite nanoparticles [14]. Magnetite $\left(\mathrm{Fe}_{3} \mathrm{O}_{4}\right)$ is the most often used magnetic material in the MNP field. It is simply produced from cheap starting materials, usually by coprecipitation, i.e., mixing $\mathrm{Fe}(\mathrm{III})$ and $\mathrm{Fe}(\mathrm{II})$. As an alternative, mere $\mathrm{Fe}(\mathrm{II})$ was used as a starting material for magnetite. In such cases, part of the $\mathrm{Fe}(\mathrm{II})$ is oxidized under reaction conditions (oxygen in the air) in an alkaline aqueous solution at low temperatures [15]. For instance, using somewhat harsher conditions, i.e., a higher temperature and longer reaction times, dodecylamine was previously applied in the presence of sodium hydroxide [16-19]. The technology starting with Fe(II) was not employed for 1-(3-aminopropyl)-imidazole (AIm) as a stabilizer or in combination with sodium hydroxide, neither at an elevated temperature nor at room temperature, and is followed up in the present work. 
We synthesized a new MNP starting from Fe(II) using AIm and hydroxide at room temperature in one approach. AIm contains two functional groups: amino and imidazole. It can be assumed that the imidazole moiety of AIm, with its strong, complex properties for metal ions [20,21], is linked to the magnetite surface, while the amino group remained untouched. Thus, it can serve as a linking point for further functionalization. This linking reaction is a straightforward and fast way to obtain functionalized magnetic nanoparticles. Poly(benzofurane-co-arylacetic acid) (PBAAA) was used for the attachment of the polymer on the preformed amino-functionalized magnetite, applying a "grafting-from" strategy. Here, the amino groups cause the opening of the lactone rings of PBAAA, which also gives evidence that imidazole is linked to the MNP surface rather than the amino groups of AIm.

\section{Materials and Methods}

\subsection{Materials}

All of the reagents used in this work were acquired from Sigma Aldrich (St. Louis, MO, USA) and Alfa Aesar by ThermoFisher Scientific (Kandel, Germany) and did not need to be further purified. PBAAA (poly(benzofurane-co-arylacetic acid)) was synthesized according to previously published protocols [22].

\subsection{Preparation of Uncovered Magnetic Nanoparticles (MNP)}

A total of $2 \mathrm{M} \mathrm{NaOH}(60 \mathrm{~mL})$ was added under vigorous stirring at room temperature to a solution formed by dissolving iron(II)chloride tetrahydrate $(5.94 \mathrm{~g}, 30 \mathrm{mmol})$ in deionized water $(150 \mathrm{~mL})$. The mixture was stirred at room temperature for $30 \mathrm{~min}$. After cooling down, the resulting black magnetic solid was magnetically separated and washed repeatedly with distilled water and acetone, yielding $2.68 \mathrm{~g}$ magnetite nanoparticles MNP.

\subsection{Preparation of Magnetic Nanoparticles Stabilized with 1-(3-Aminopropyl)imidazole (MNP-AIm_1)}

1-(3-aminopropyl)imidazole AIm $(12.5 \mathrm{~mL}, 100 \mathrm{mmol}$ ) was added under vigorous magnetic stirring at room temperature to a solution formed by dissolving iron(II)chloride tetrahydrate $(0.8 \mathrm{~g}, 4 \mathrm{mmol})$ in deionized water $(100 \mathrm{~mL})$. The mixture was allowed under magnetic stirring at room temperature for $30 \mathrm{~min}$. Afterwards, the functionalized magnetic nanoparticles of MNP-AIm_1 were separated magnetically and rinsed with water and acetone. After drying, 0.54 g of MNP-AIm_1 was obtained as black magnetic material.

2.4. Preparation of Magnetic Nanoparticles Stabilized with 1-(3-Aminopropyl)imidazole (MNP-AIm_2 in the Presence of Aqueous Sodium Hydroxide)

A total of $2 \mathrm{M} \mathrm{NaOH}(60 \mathrm{~mL})$ was added to a solution of iron(II)chloride tetrahydrate $(5.94 \mathrm{~g}, 30 \mathrm{mmol})$ in deionized water $(150 \mathrm{~mL})$, followed by the addition of AIm $(6.25 \mathrm{~mL}$, $50 \mathrm{mmol}$ ) at room temperature. After $30 \mathrm{~min}$, the resulting black magnetic solid was magnetically separated by an external magnet. Next, the solid black material was washed successively with distilled water and acetone, yielding $2.88 \mathrm{~g}$ MNP-AIm_2.

\subsection{Poly(benzofuran-co-arylacetic acid) Linkage on the Amino-Functionalized Magnetic Nanoparticles Surface (MNP-AIm_PAAA)}

The magnetic nanoparticles MNP-AIm_1 $(0.1 \mathrm{~g})$ were dispersed in distilled water (30 mL), and poly(benzofuran-co-arylacetic acid) $(0.2 \mathrm{~g})$ was added to the mixture. The reaction mixture was stirred at room temperature for $24 \mathrm{~h}$. The polymer-covered magnetic nanoparticles MNP-AIm_PAAA were separated with an external magnet. After successive washings with methanol and acetone, the magnetic nanostructures of MNP-AIm_PAAA were dried in the oven at $50^{\circ} \mathrm{C}$ and further analyzed.

\subsection{Instrumentation}

FTIR (Fourier transform infrared spectroscopy) spectra $\left(400-4000 \mathrm{~cm}^{-1}\right)$ were obtained on a pressed tablet prepared from magnetic powder enclosed in $\mathrm{KBr}$, with a JASCO FTIR-6100 spectrophotometer (JASCO Deutschland GmbH, Pfungstadt, Germany). XRD 
data were collected using a Smart Lab Rigaku diffractometer with a graphite monochromator for $\mathrm{Cu}-\mathrm{K} \alpha$ radiation $(\lambda=1.54 \AA$ ) at room temperature. Hitachi H9000NAR (Hitachi Ltd., Tokyo, Japan) and 1010 JEOL transmission electron microscopes (JEOL Ltd., Tokyo, Japan) were used to establish the MNP's morphology. The chemical surface of the functionalized magnetic nanoparticles was studied using X-ray photoelectron spectroscopy (XPS). An XPS spectrometer (SPECS, Berlin, Germany) containing a dual-anode X-ray source $\mathrm{Al} / \mathrm{Mg}$, a PHOIBOS 150 2D CCD hemispherical energy analyzer, and a multi-channeltron detector was employed to record the XPS spectra at a vacuum of 1.9109 torrs. The XPS spectra were acquired at $30 \mathrm{eV}$ pass energy, with a $0.5 \mathrm{eV} /$ step, using an $\mathrm{AlK} X$-ray source $(1486.6 \mathrm{eV})$ at a power of $200 \mathrm{~W}$. Individual element high-resolution images were obtained by collecting 10-15 scans at $30 \mathrm{eV}$ pass energy with $0.1 \mathrm{eV} /$ step. A Cryogenic Vibrating Sample Magnetometer (Cryogenic Ltd., London, UK) was applied to perform magnetic measurements at room temperature. Thermogravimetry measurements were achieved in the air using TA Instruments SDT Q 600 equipment (TA Instruments Inc., New Castle, DE, USA) at a heating rate of $10^{\circ} \mathrm{C} \mathrm{min}^{-1}$ within $30^{\circ} \mathrm{C}$ to $800{ }^{\circ} \mathrm{C}$.

\section{Results and Discussion}

\subsection{Synthesis of Magnetic Nanostructure MNP@AIm}

We successfully proved that the methodology of using just $\mathrm{FeCl}_{2}$ without any $\mathrm{Fe}(\mathrm{III})$ source can also be applied in the straightforward synthesis of functionalized magnetite nanoparticles MNP-AIm when AIm, as a stabilizer, is used. Envisaging synthesis at a large scale, it would be advantageous to reduce the quantity of AIm. We first checked that sodium hydroxide could be used as a base in the preparation of magnetic nanoparticles, starting from $\mathrm{Fe}(\mathrm{II})$. In fact, MNP was obtained at room temperature after $30 \mathrm{~min}$ (Scheme 1). Taking this into consideration, we used the less cost-intensive $\mathrm{NaOH}$ as a base and a smaller quantity of AIm and thus obtained MNP-AIm_2 after $30 \mathrm{~min}$ at room temperature. All products appeared as magnetic black solids and were quickly separated by an external magnet and purified by washing with water and acetone. They were investigated through the several previously mentioned methods.

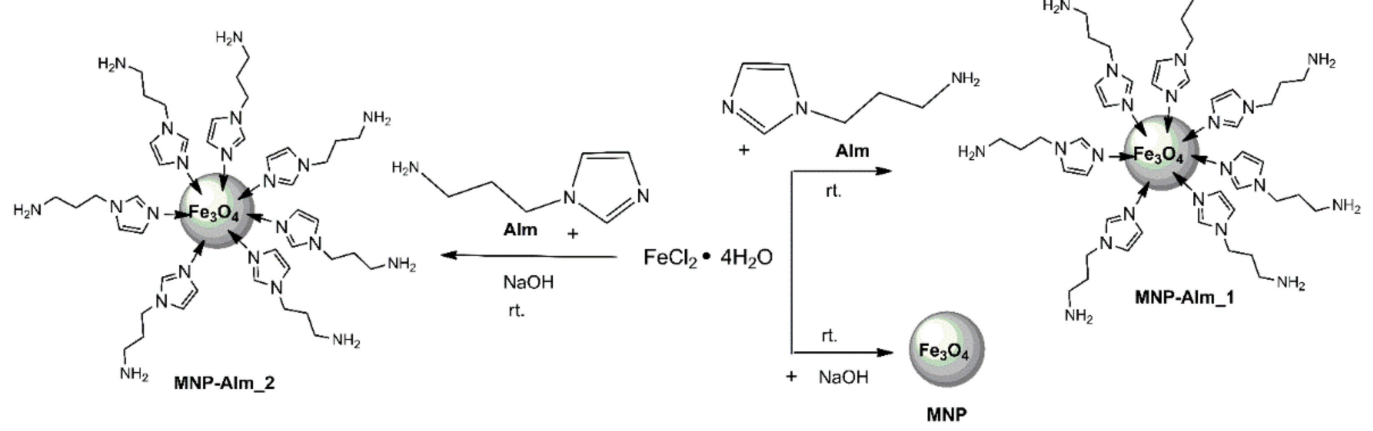

Scheme 1. Preparation of magnetite nanoparticles MNP, MNP-AIm_1, and MNP-AIm_2.

Due to the presence of the amino group in AIm molecules on the magnetic nanoparticles surface, one can expect that MNP-AIm_1 serves as a good platform for further immobilization of polymers or other molecules with relevance for practical applications. We demonstrated this potential by linking poly(benzofurane-co-arylacetic acid) PBAAA (Scheme 2). This polymer can easily be synthesized in a poly-Friedel-Crafts alkylation by controlled heating of 4-hydroxymandelic acid. It contains lactones, esters, and carboxyl groups. The preparation of magnetic nanoparticles coated with PBAAA consists of two steps: (i) synthesis of amino-functionalized magnetite nanoparticles through a straightforward method; (ii) link of poly(benzofurane-co-arylacetic acid) on the surface of these amino-functionalized MNPs, wherein the amino groups open lactone rings, resulting in amide moieties. The amino groups alone caused the opening of the lactone rings in PBAAA, 
giving evidence that imidazole is linked to the MNP surface rather than the amino groups of AIm.

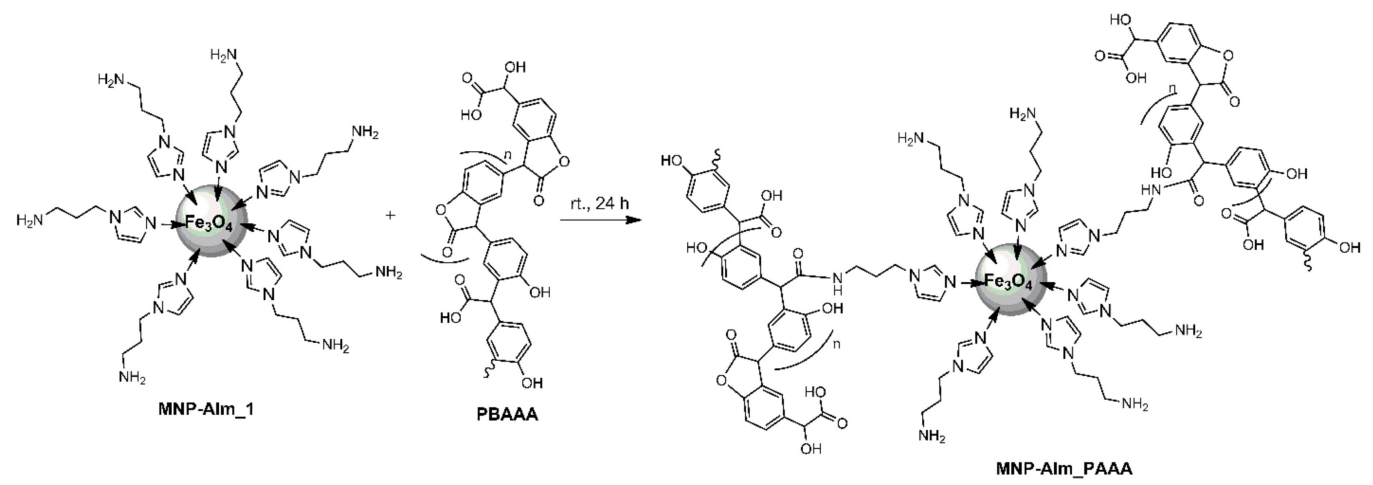

Scheme 2. Coating of magnetite nanoparticles MNP-AIm_1 with poly(benzofuran-co-arylacetic acid) PBAAA.

\subsection{Characterization of Magnetic Nanostructure MNP@AIm \\ 3.2.1. Infrared Spectroscopy}

Figure 1 shows noteworthy changes in the FTIR spectra of coated magnetite MNPAIm_1 and the MNP-AIm_2 FTIR spectra compared to the uncoated magnetite MNP. In all FTIR spectra, the intensive peak of the specific absorption band of the Fe-O bond is roughly at the same wavelength, namely $580 \mathrm{~cm}^{-1}$, and is associated with the stretching vibration of the tetrahedral groups from $\mathrm{Fe}_{3} \mathrm{O}_{4}$. In the MNP-AIm_1 (where only AIm was used for preparation) FTIR spectrum, the absorption bands ascribed to the AIm moiety are slightly more intense than MNP-AIm_2. The $-\mathrm{NH}_{2}$ group possesses six normal modes. Not all of them are visible in the present FTIR spectra, the antisymmetric and symmetric stretching modes are at $3586 \mathrm{~cm}^{-1}$, and the absorption peak from $1522 \mathrm{~cm}^{-1}$ belongs to the in-plane bending vibration of the amine group. The scaled frequency for $-\mathrm{NH}_{2}$ rocking mode is assigned at $1107 \mathrm{~cm}^{-1}$, and the $-\mathrm{NH}_{2}$ wagging mode appears at $890 \mathrm{~cm}^{-1}$. In general, the imidazole ring-stretching vibrations have five normal modes. Two frequencies are allocated between 1490 and $1660 \mathrm{~cm}^{-1}$, while the other three are between $1300-1000 \mathrm{~cm}^{-1}$. At $1660 \mathrm{~cm}^{-1}$, the adsorption band that is attributed to the $\mathrm{C}=\mathrm{N}$ stretching mode can be found. The absorption band from $1440 \mathrm{~cm}^{-1}$ ascribes the N-H in-plane bending mode and the methylene scissoring from the imidazole ring. The scaled frequencies, which are associated with the $\mathrm{C}-\mathrm{H}$ and $=\mathrm{C}-\mathrm{N}$ bond in-plane bending modes, are found at $1235 \mathrm{~cm}^{-1}$. The C-C stretching frequency is located at $1102 \mathrm{~cm}^{-1}$ and the C-N stretching mode is appointed at $950 \mathrm{~cm}^{-1}$. The last two mentioned bonds belong to the propylamine chain. The presence of the absorption bands specific to magnetite and those of the AIm component in both FTIR spectra provide evidence of the formation of functional magnetite in MNPAIm_1, as well as in MNP-AIm_2.

Figure 2 reveals important changes in the FTIR spectrum of amino-imidazole functionalized magnetic nanoparticles MNP-AIm_1 and the magnetic nanostructures of MNPAIm_PAAA. The FTIR of MNP AIm_PAAA included specific absorption bands for both the magnetite and the polymer, demonstrating the attachment of the polymer to the MNP surface. The absorption band indicating the covalent bonding of the polymer on the MNPAIm_1 surface was located at $1800 \mathrm{~cm}^{-1}$ for MNP-AIm_PAAA and was assigned to the $\mathrm{C}=\mathrm{O}$ band from the benzofuranone ring. The intensity of the benzofuranone $\mathrm{C}=\mathrm{O}$ band at $1800 \mathrm{~cm}^{-1}$ was dramatically reduced compared to the non-linked PBAAA, demonstrating the transformation of the lactone into amide moieties, i.e., a covalent bond. The amide bond has a broad absorption band at $1620 \mathrm{~cm}^{-1}$. The shoulder at $1730 \mathrm{~cm}^{-1}$ in the MNPAIm_PAAA spectrum can be attributed to the carboxyl moiety. The two weak absorption bands at 2856 and $2921 \mathrm{~cm}^{-1}$ in the FTIR spectra of the polymer PBAAA and the magnetic nanostructure AIm_PAAA, respectively, were ascribed to the aliphatic $\mathrm{CH}$-groups and the stretching-mode band for the $=\mathrm{C}-\mathrm{H}$ bond. The split in the FTIR spectrum of MNP- 
AIm_PAAA at $585 \mathrm{~cm}^{-1}$ and $630 \mathrm{~cm}^{-1}$ wave numbers was due to the principal effect of the nanosized iron oxide particles, where a part of the polymer is interacting with the MNP's surface. The unbinding of the many bonds from the surface atoms resulted in the rearrangement of localized electrons on the surface of the magnetite nanoparticles [23].

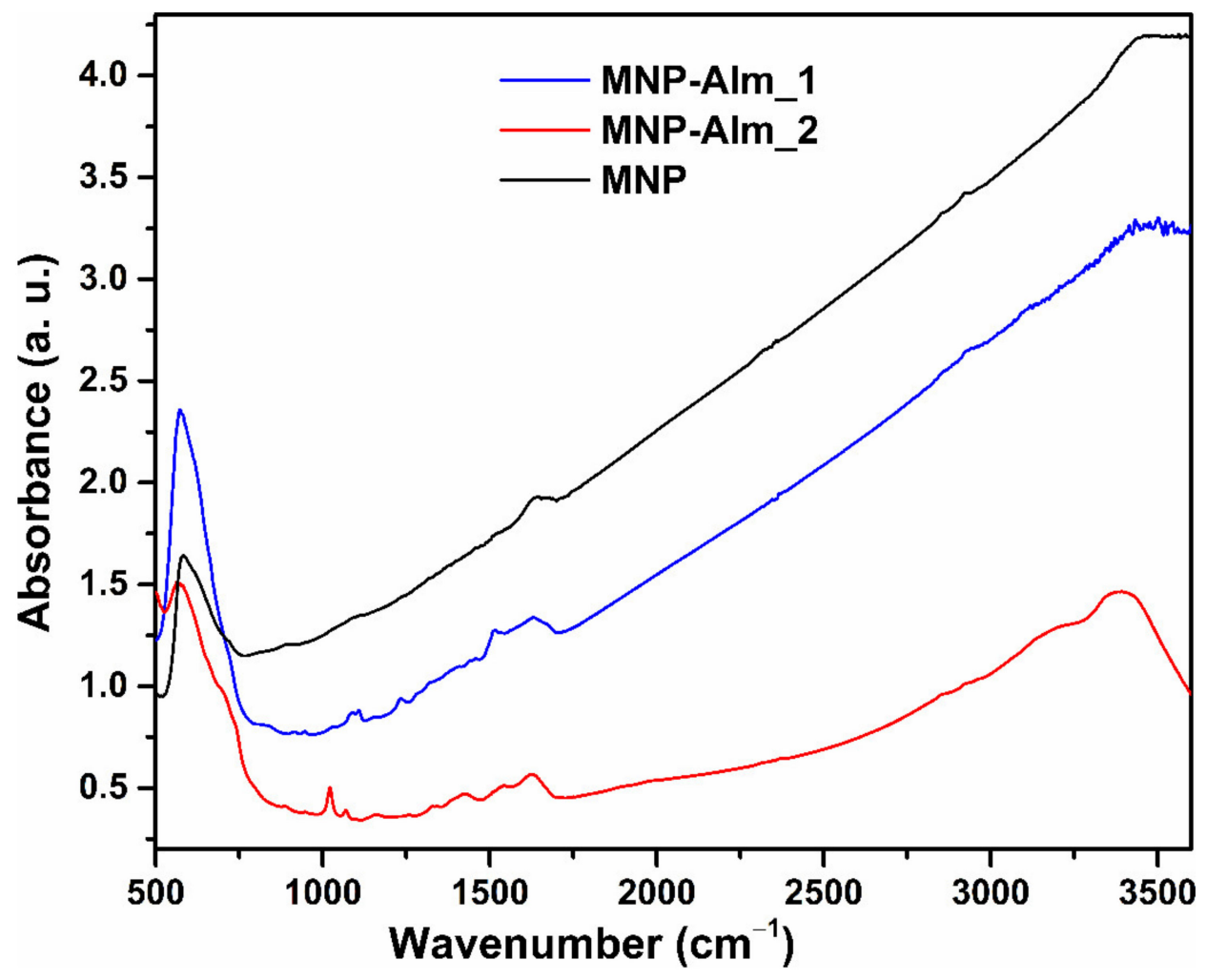

Figure 1. FTIR spectra of uncoated MNP, MNP-AIm_1, and MNP-AIm_2 in KBr pellets.

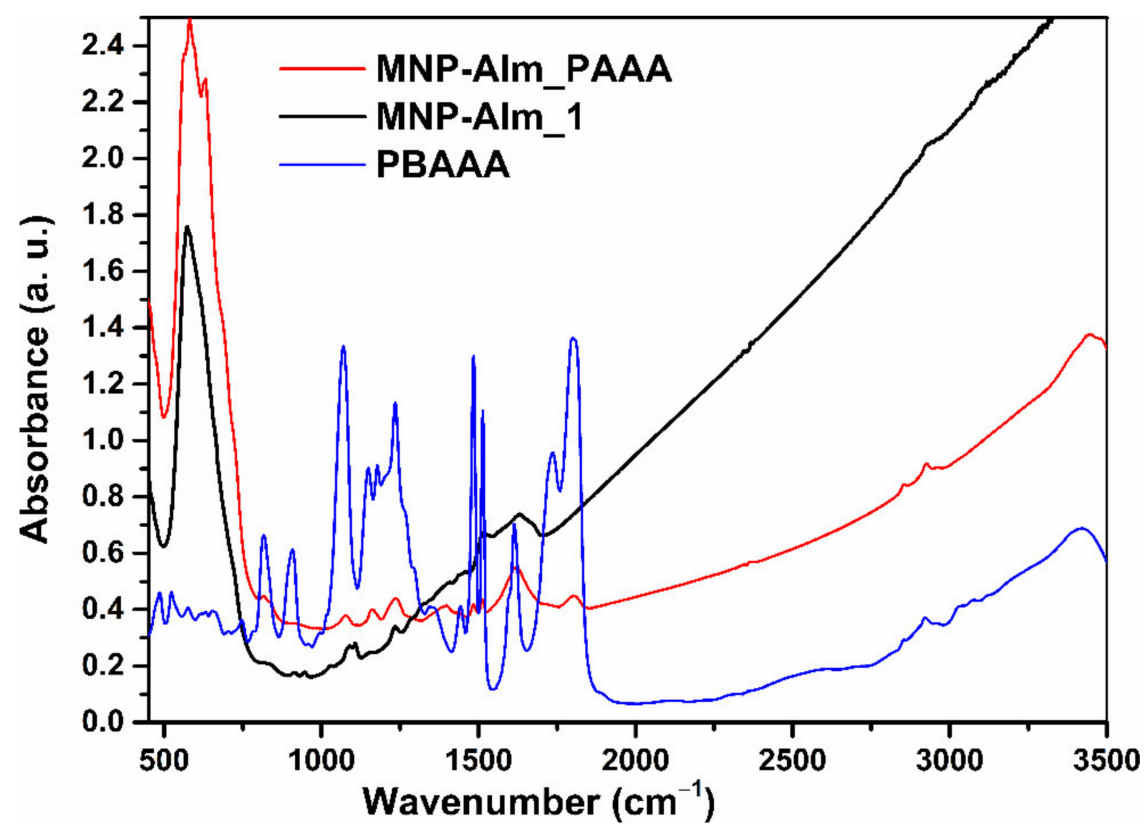

Figure 2. FTIR spectra of functionalized magnetic nanoparticles MNP-AIm_1, polymer PBAAA and magnetic nanostructure MNP-AIm_PAAA. 


\subsubsection{X-ray Powder Diffraction}

The X-ray diffractograms of MNP, MNP-AIm_1, and MNP-AIm_2 were measured at room temperature and are presented in Figure 3. The diffraction peaks of (111), (220), (311), (400), (422), (511), (440), and (533) reflect the magnetite crystal with a cubic spinel structure. Considering all the results achieved in XRD using the Scherrer equation, a non-significant change was observed regarding the crystallite size. The uncovered MNP was obtained with a crystallite size of $34.5 \mathrm{~nm}$, while the MNP-AIm_1 synthesized only in the presence of AIm, which had a crystallite size of $30 \mathrm{~nm}$. A crystallite size of $34.2 \mathrm{~nm}$ was observed in MNP-AIm_2, which was synthesized in the presence of AIm and $\mathrm{NaOH}$.

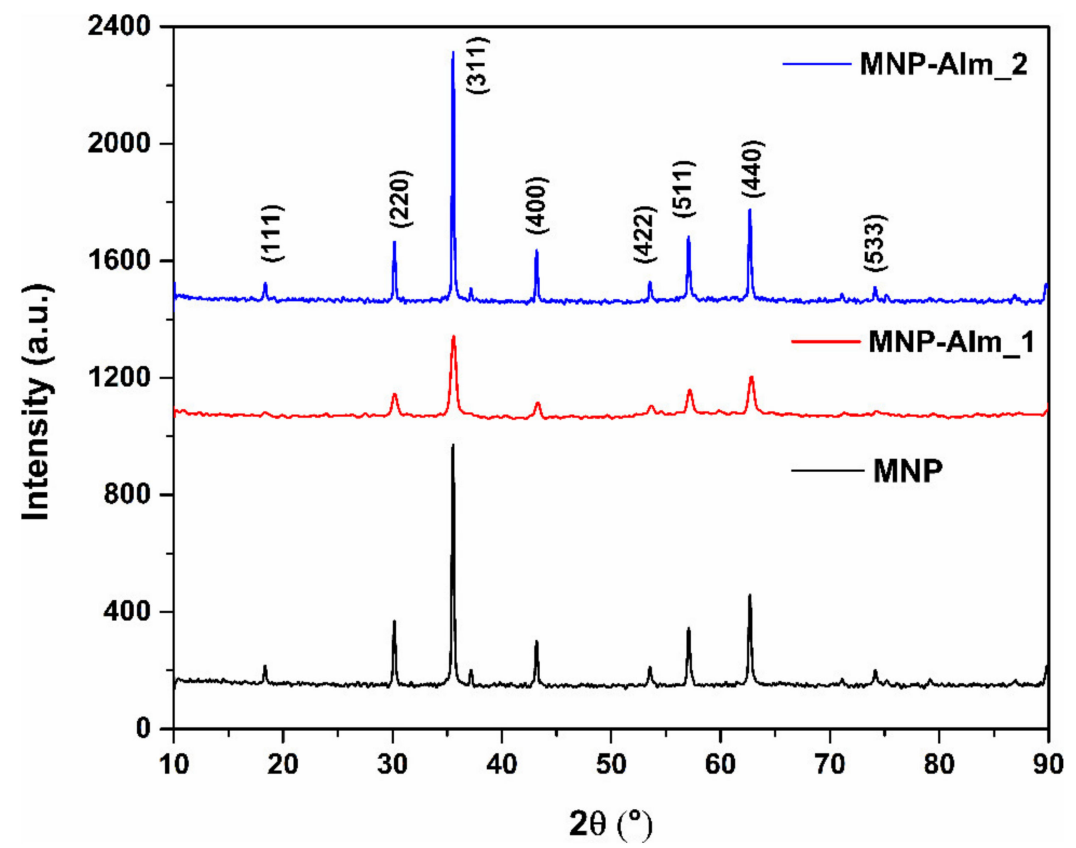

Figure 3. X-ray diffraction (XRD) patterns of magnetic nanoparticles MNP, MNP-AIm_1, and MNPAIm_2.

\subsubsection{Transmission Electron Microscopy}

TEM investigations of MNP, MNP-AIm_1, and MNP-AIm_2 (Figures 4 and 5) revealed different morphologies. The TEM images of MNP (Figure 4a) and MNP-AIm_2 (Figure 4b) form suspensions with partial aggregation, but in the case of MNP-AIm_1 (Figure 5a), TEM images reveal an excellent dispersion of the magnetite nanoparticles.
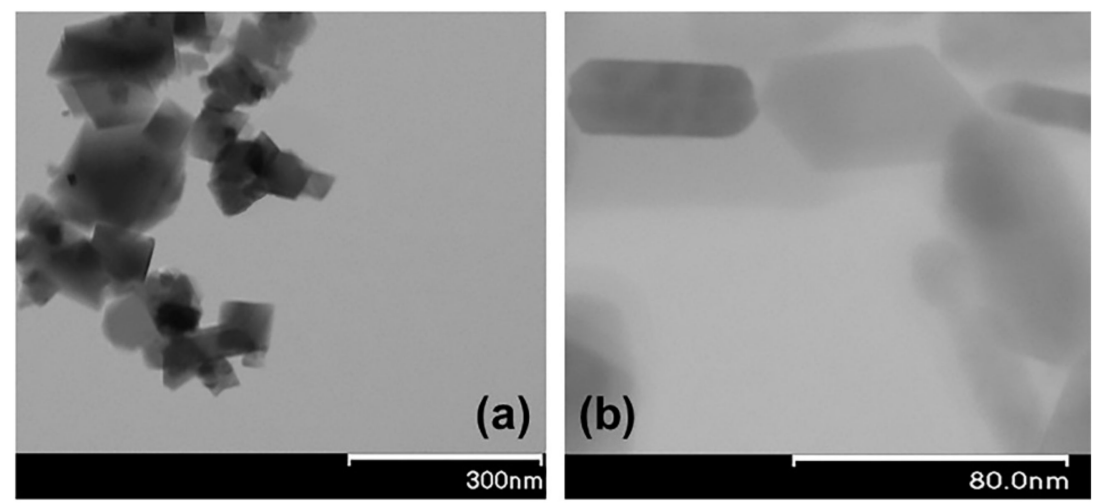

Figure 4. TEM image of uncovered MNP (a) and stabilized magnetic nanoparticles MNP-AIm_2 (b), obtained by depositing $10 \mu \mathrm{L}$ of a suspension of each sample onto a copper grid coated with a thin carbon film and drying it. 

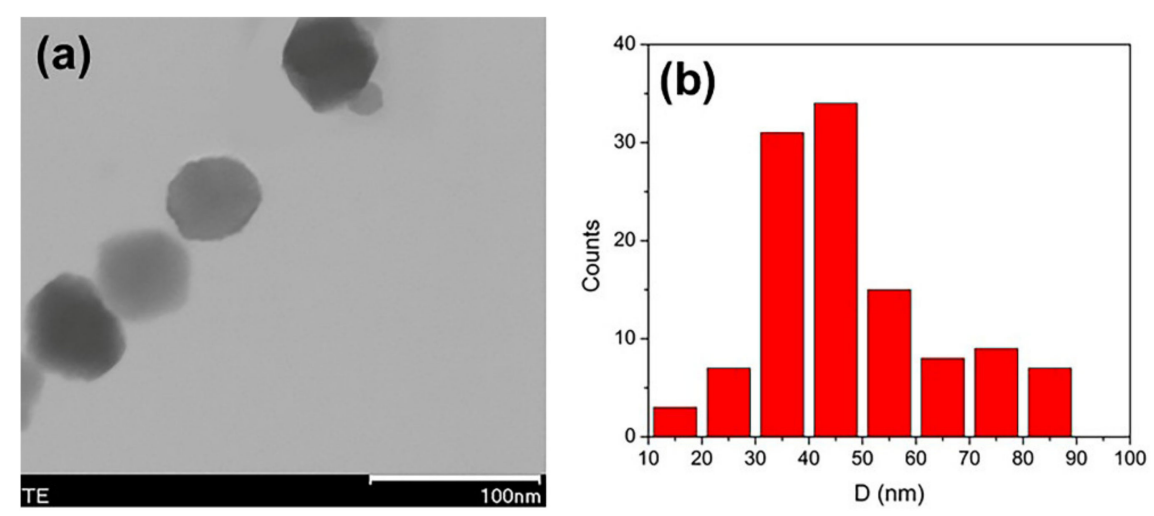

Figure 5. TEM image of stabilized magnetic nanoparticles MNP-AIm_1 (a) obtained by depositing $10 \mu \mathrm{L}$ of a suspension of each sample onto a copper grid coated with a thin carbon film and drying it and size distribution (diameter) of the magnetic nanoparticles MNP-AIm_1 based on TEM measurements (b).

The synthesis conditions strongly influence the morphology of the magnetic nanoparticles: MNP contained spherical and cubic nanoparticles (Figure 4a), MNP-AIm_1 displayed spherical nanoparticles with a mean diameter $\mathrm{D}=45 \mathrm{~nm}$ (Figure 5). In comparison, rodlike nanoparticles with a mean length of $70 \mathrm{~nm}$ characterized the sample MNP-AIm_2 (Figure $4 \mathrm{~b}$ ). It seems that the synthesis in the presence of $\mathrm{NaOH}$, as used in the preparation of MNP-AIm_2, favored the appearance of the nanoparticles' morphologies differently from the spherical ones found in MNP-AIm-1.

\subsubsection{X-ray Photoelectron Spectroscopy}

The XPS survey spectra showed all element peaks of the MNP, MNP-AIm_1, and MNP-AIm_2 samples (Figure 6).

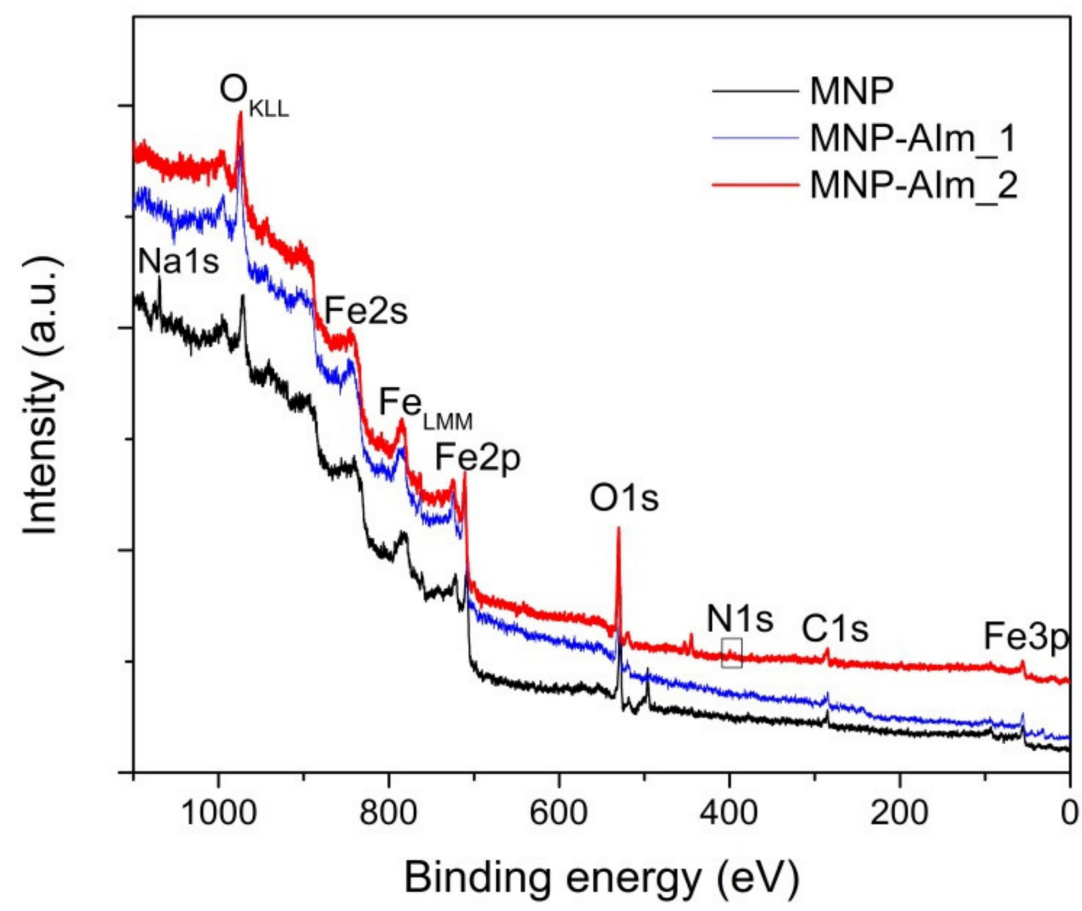

Figure 6. XPS survey spectra for MNP, MNP-AIm_1 and MNP-AIm_2.

The high-resolution XPS spectrum of Fe2p for MNP shown in Figure 7 included the doublet Fe2p3/2 and Fe2p1/2. The components corresponding to $\mathrm{Fe}^{2+}$ octahedral, $\mathrm{Fe}^{3}+$ octahedral, and $\mathrm{Fe}^{3+}$ tetrahedral, respectively, and the satellites provided the best 
fit for the Fe2p spectrum [24,25]. We determined the ratio of the atomic concentration $\mathrm{Fe}^{3+} / \mathrm{Fe}^{2+}=2.3$ from the $\mathrm{Fe}^{3+}$ and $\mathrm{Fe}^{2+}$ peak areas; this value is close to that expected for magnetite. Similar XPS spectra of Fe2p were obtained for the samples MNP-AIm_1, MNP-AIm_2, and MNP-AIm_PAAA.

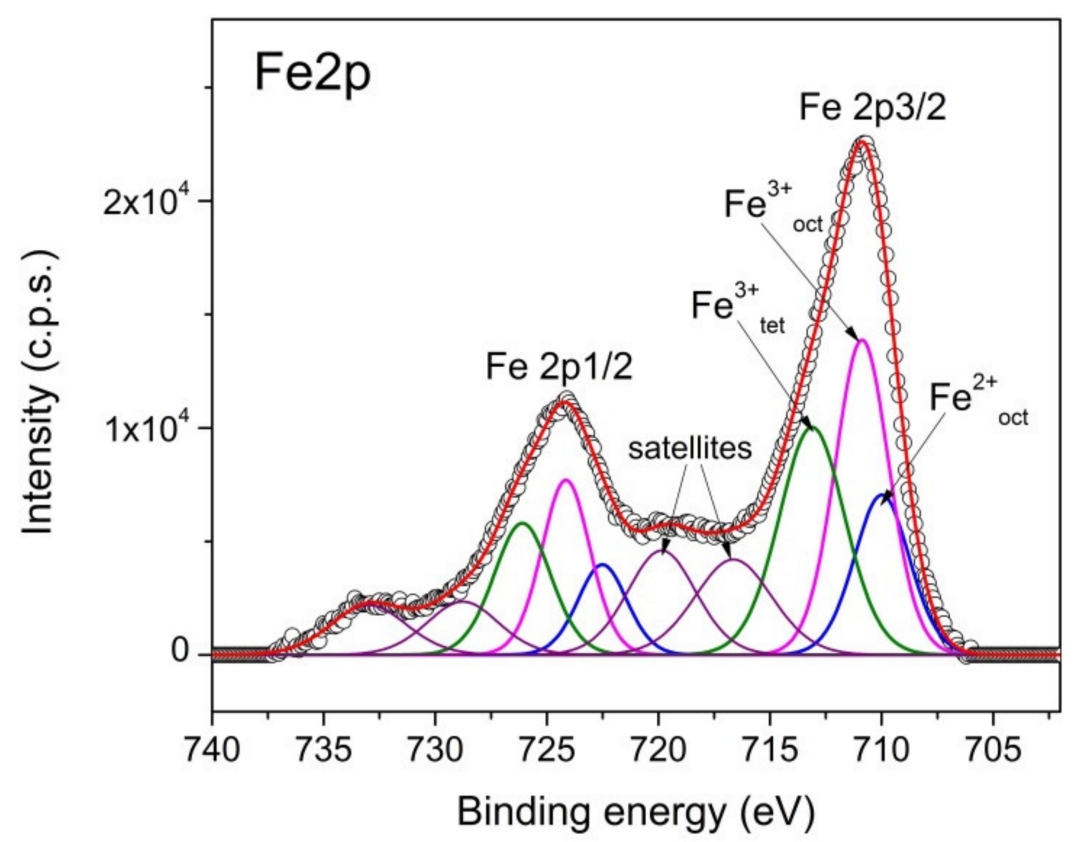

Figure 7. High-resolution XPS spectrum of Fe2p core levels for MNP.

In Figures 8 and 9, the high-resolution XPS spectra of the C1s, O1s, N1s, and Na1s core levels for samples MNP-AIm_1 and MNP-AIm_2, respectively, are presented. The deconvolution of the XPS peaks of the C1s, O1s, and N1s in Figures 8 and 9 showed similar peaks.
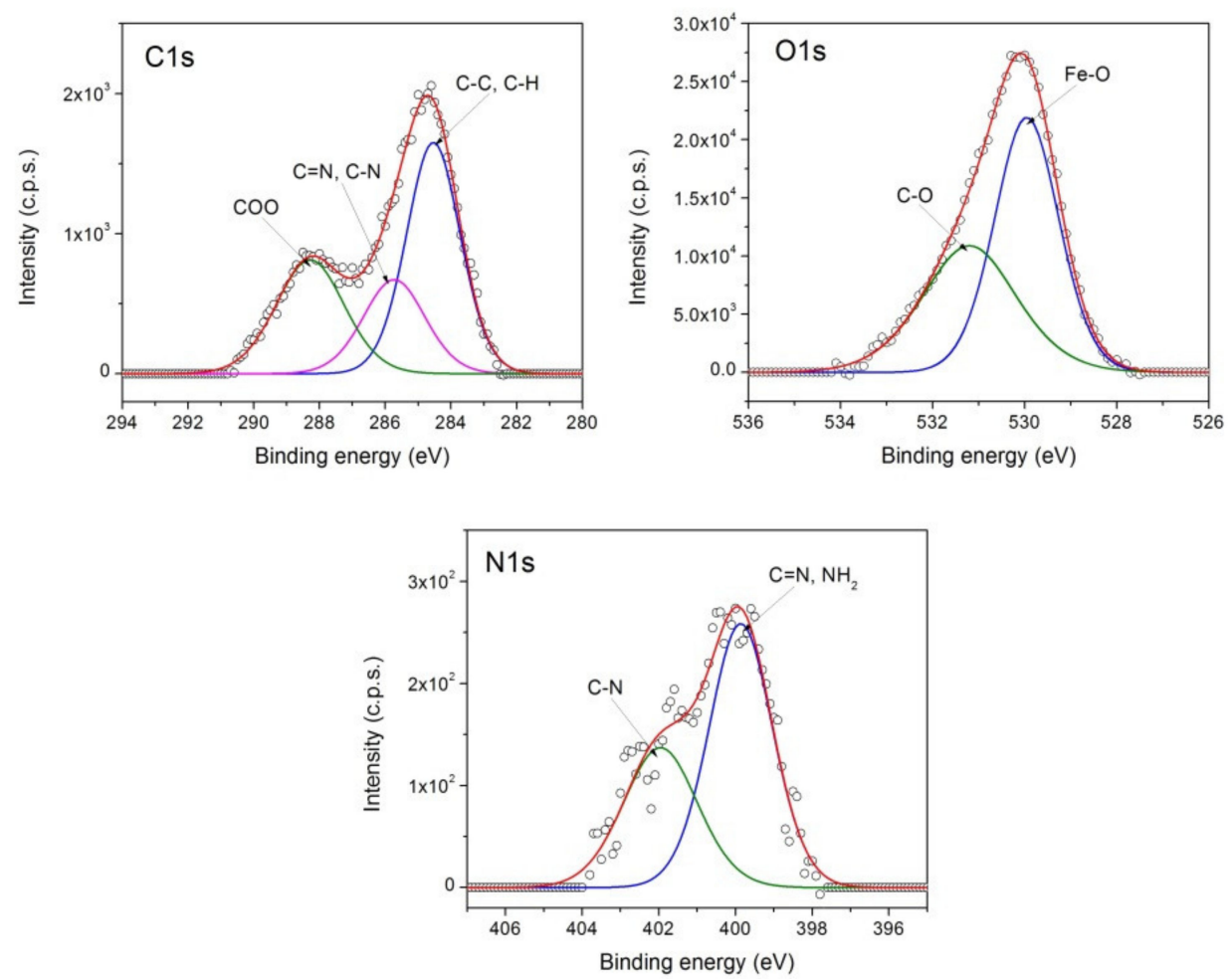

Figure 8. High-resolution XPS spectra of C1s, O1s and N1s core-levels for MNP-AIm_1. 

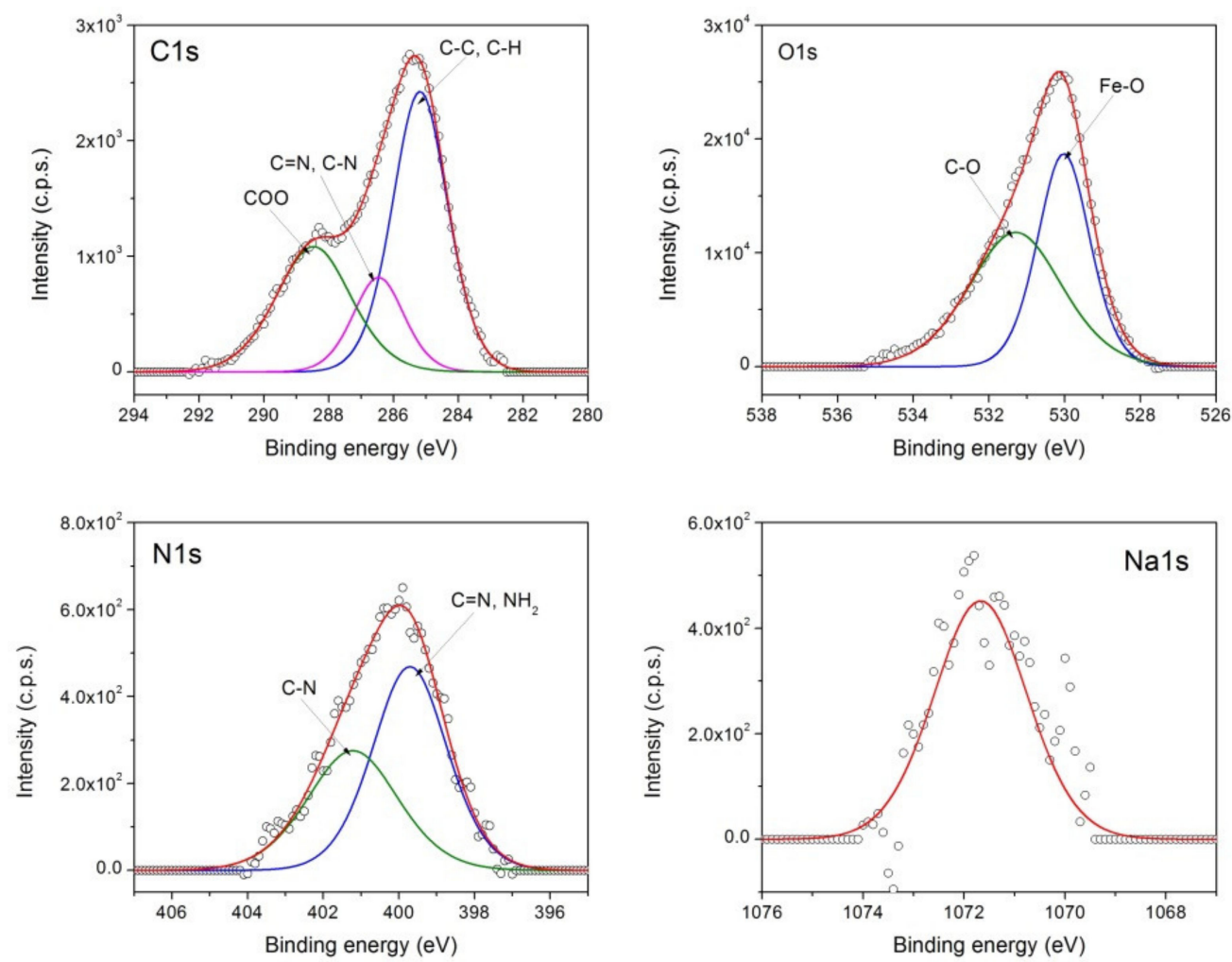

Figure 9. High-resolution XPS spectra of C1s, O1s, N1s and Na1s core-levels for MNP-AIm_2.

The best fit for the $\mathrm{C} 1 \mathrm{~s}$ spectrum was obtained with three components assigned to the carbon atoms from C-C, $\mathrm{CH}(284.5 \mathrm{eV}), \mathrm{C}=\mathrm{N}, \mathrm{C}-\mathrm{N}(285.7 \mathrm{eV})$ groups characteristic to 1-(3-aminopropyl)imidazole, and $\mathrm{COO}(288.3 \mathrm{eV})$ due to environmental contamination. The O1s spectrum exhibited two components: one located at $530 \mathrm{eV}$ ascribed to Fe-O from magnetite and the other located at $531 \mathrm{eV}$ from $\mathrm{C}-\mathrm{O}$, which are also associated with contamination. The N1s spectrum showed two peaks assigned to $\mathrm{C}=\mathrm{N}, \mathrm{NH}_{2}(399.8 \mathrm{eV})$, and C-N $(401.9 \mathrm{eV})$, which are characteristic of imidazole [26].

The XPS spectra from Figures 8 and 9 demonstrate the coating of the magnetite nanoparticles with 1-(3-aminopropyl)imidazole. Moreover, the sample MNP-AIm_2 contained $\mathrm{Na}$, which was adsorbed in the sample due to the reaction conditions.

The atomic concentrations (\%) calculated from the XPS spectra for MNP-AIm_1 and MNP-AIm_2 given in Table 1 highlights an increase in the C concentration for the sample MNP-AIm_2 as compared with MNP-AIm_1, indicating a higher degree of coating of the nanoparticles with 1-(3-aminopropyl)imidazole MNP-AIm_2.

Table 1. The atomic concentrations (\%) were calculated from XPS spectra for MNP-AIm_1, MNPAIm_2 and MNP-AIm_PAAA.

\begin{tabular}{cccccc}
\hline \multirow{2}{*}{ Sample } & \multicolumn{5}{c}{ Atomic Concentrations (\%) } \\
\cline { 2 - 6 } & Fe & $\mathbf{C}$ & $\mathbf{O}$ & $\mathbf{N}$ & $\mathbf{N a}$ \\
\hline MNP-AIm_1 & 46.95 & 11.891 & 40.326 & 0.833 & - \\
MNP-AIm_2 & 41.253 & 16.213 & 40.437 & 1.841 & 0.256 \\
MNP-AIm_PAAA & 22.870 & 30.242 & 45.478 & 1.41 & - \\
\hline
\end{tabular}

The high-resolution XPS spectra of the C1s, O1s, and N1s core levels for the sample MNP-AIm_PAAA are shown in Figure 10. The deconvolution of the C1s spectrum from Figure 10 shows four peaks characteristic of the polymer coating layer: $\mathrm{C}-\mathrm{C}, \mathrm{C}-\mathrm{H}(284.9 \mathrm{eV})$; $\mathrm{C}=\mathrm{N}, \mathrm{C}-\mathrm{N}, \mathrm{C}-\mathrm{O}(286.2 \mathrm{eV}) ; \mathrm{C}=\mathrm{O}(288.32 \mathrm{eV}) ; \mathrm{O}-\mathrm{C}=\mathrm{O} 289.28 \mathrm{eV})$. The O1s spectrum contained three components: $530.09 \mathrm{eV}(\mathrm{Fe}-\mathrm{O}) ; 531.35 \mathrm{eV}(\mathrm{C}-\mathrm{O}, \mathrm{N}-\mathrm{C}=\mathrm{O}) ; 533.07 \mathrm{eV}(\mathrm{O}-\mathrm{C}=\mathrm{O})$. The best 
fit of the N1s spectrum from Figure 10 was achieved with two components ascribed to $\mathrm{C}=\mathrm{N}(399.68 \mathrm{eV})$ and $\mathrm{C}-\mathrm{N}(401 \mathrm{eV})$; the intensity ratio of these components was different from those from Figures 8 and 9 due to the poly(benzofurane-co-arylacetic acid) coupling on the surface of the amino-functionalized magnetic nanoparticles.
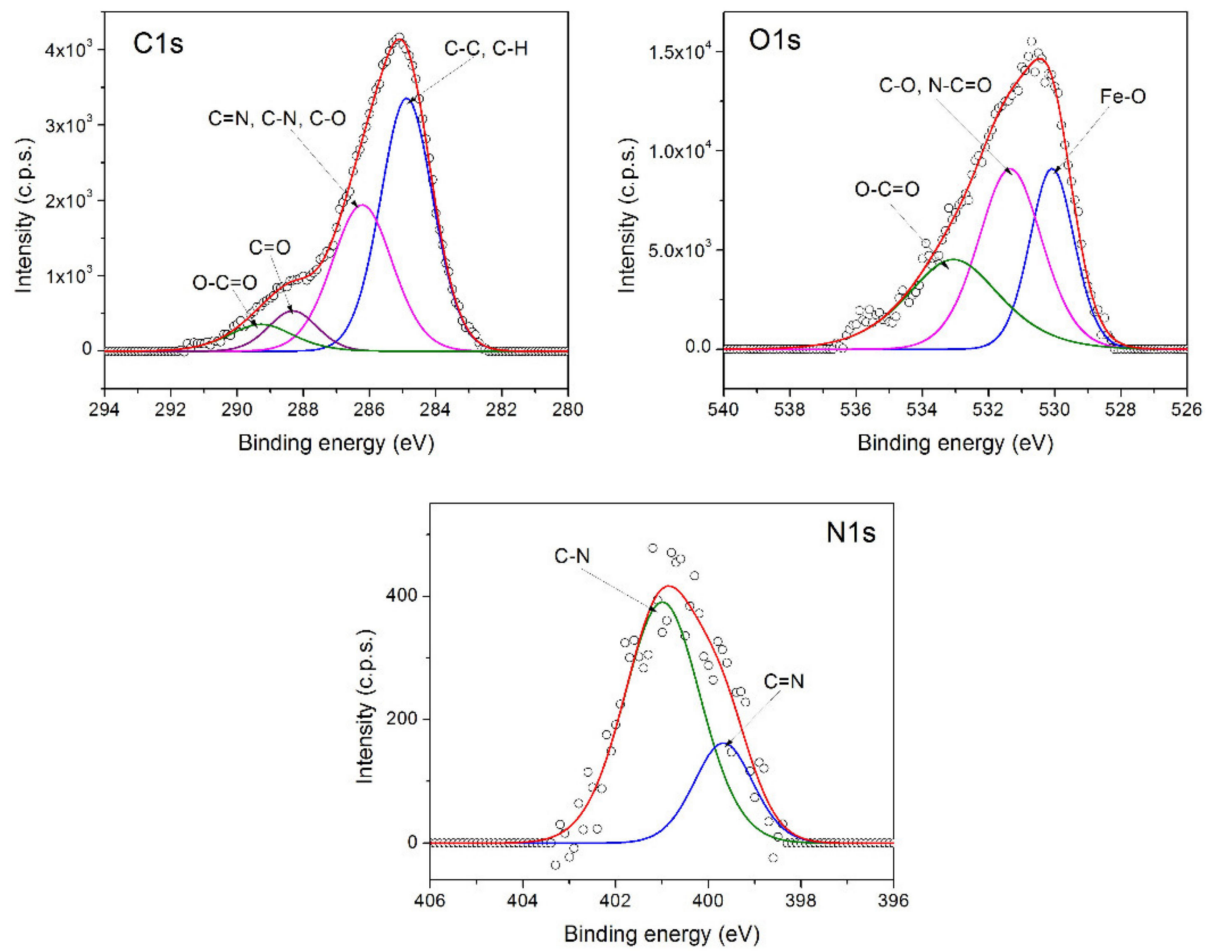

Figure 10. High-resolution XPS spectra of C1s, O1s and N1s core-levels of MNP-AIm_PAAA.

A comparison of the atomic concentrations (\%) calculated from the XPS spectra (Table 1) shows a high C concentration increase for the sample MNP-AIm_PAAA, evidence of the polymer coating of functionalized magnetic nanoparticles.

\subsubsection{Magnetic Properties}

The magnetization curves for MNP, for functionalized nanoparticles MNP-AIm_1, MNP-AIm_2 and polymer-coated nanoparticles MNP-AIm_PAAA are shown in Figure 11. These samples featured astonishingly high saturation magnetization values and low coercivity (Table 2).

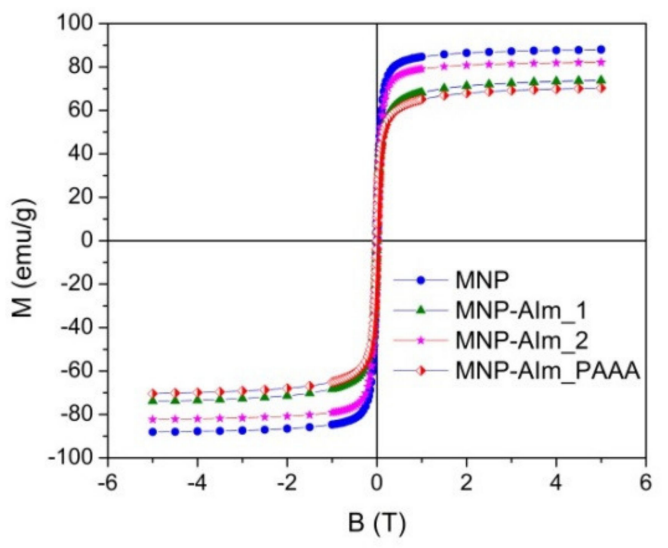

(a)

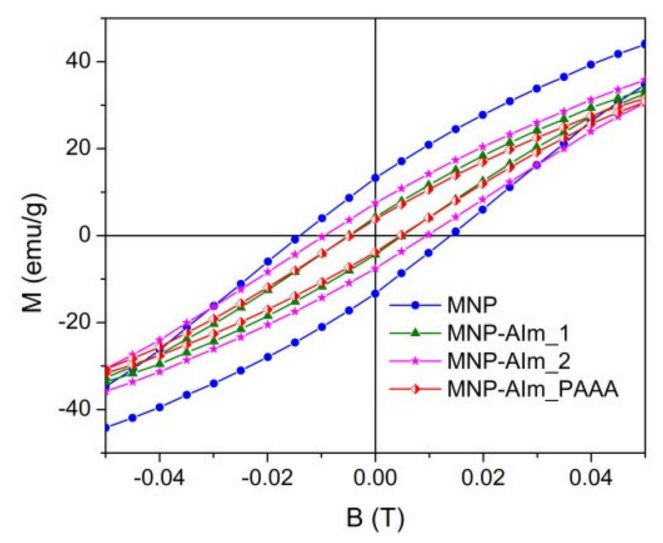

(b)

Figure 11. The magnetization (a) vs. applied magnetic field (b) of uncoated MNP and magnetic nanostructures MNP-AIm_1, MNP-AIm_2 and MNP-AIm_PAAA at room temperature. 
Table 2. The saturation magnetization $\left(\mathrm{M}_{\mathrm{S}}\right)$ and coercivity $\left(\mathrm{B}_{\mathrm{c}}\right)$ for the samples MNP, MNP-AIm_1, MNP-AIm_2 and MNP-AIm_PAAA.

\begin{tabular}{ccccc}
\hline Sample & MNP & MNP-AIm_1 & MNP-AIm_2 & MNP-AIm_PAAA \\
\hline $\mathrm{M}_{\mathrm{S}}(\mathrm{emu} / \mathrm{g})$ & 89 & 75.4 & 82.8 & 71.8 \\
\hline $\mathrm{B}_{\mathrm{c}}(\mathrm{T})$ & 0.01 & 0.005 & 0.01 & 0.005 \\
\hline
\end{tabular}

The higher saturation magnetization value for MNP-AIm_2 (82.8 emu/g) as compared to the one for MNP-AIm_1 $(75.4 \mathrm{emu} / \mathrm{g})$ was due to the difference in crystallite sizes.

\subsubsection{Thermogravimetric Analyses of Magnetic Nanoparticles}

The Thermogravimetric Analyses (TGA) curves (Figure 12) demonstrate that MNPAIm_2 contains more organic coating than MNP-AIm_1, thus confirming the conclusions drawn from the FTIR spectroscopy and XPS (see above). The mass increase seen in the uncoated MNP sample was due to the oxidation of magnetite ( $\mathrm{FeO} \cdot \mathrm{Fe}_{2} \mathrm{O}_{3}$ ) to iron(III) oxide $\mathrm{Fe}_{2} \mathrm{O}_{3}$ due to the thermal degradation that occurred in the air's atmosphere. Because the oxidation process is exothermic, the kinetic rate of the oxidation reaction of magnetite to hematite increased as the temperature rose. Therefore, the amount of oxygen produced by the $\mathrm{Fe}(\mathrm{II})$ to $\mathrm{Fe}(\mathrm{III})$ oxidation process increased proportionally with the amount of $\mathrm{Fe}$ (II) initially present $[27,28]$.

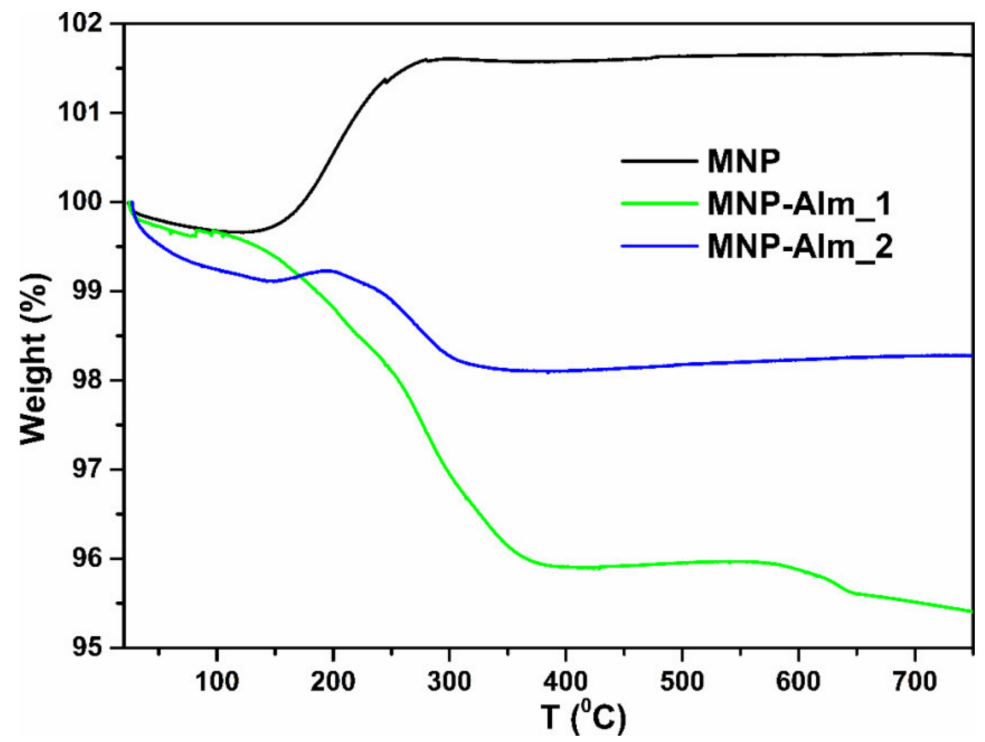

Figure 12. TGA curve of uncovered magnetic nanoparticles MNP, MNP-AIm_1, and MNP-AIm_2.

\section{Conclusions}

A remarkably straightforward synthesis of functionalized MNP was found by treating aqueous solutions of $\mathrm{FeCl}_{2}$ with 1-(3-aminopropyl)imidazole in the air at room temperature. The structural characterization of the newly functionalized magnetic nanoparticles was carried out using FTIR and XPS spectroscopy. AIm ensures, on the one hand, an excellent fixation on the surface of MNPs through the imidazole ring, which is a powerful complexing agent, and, on the other side, offers functionalization with the amino groups of these nanoparticles, and all of this occurs in a short reaction time and at low cost. X-ray powder diffraction (XRD) and TEM data were used to examine the form and size of the as-prepared magnetite nanoparticles, and the drawn conclusion is that depending on the chosen base, we prepared nanoparticles with a defined, spherical, cubic or rod-like shape. The magnetization measurements recorded at room temperature were in good agreement with the data computed using the newly developed core-shell model, confirming the superparamagnetism of the synthesized MNPs. The high saturation magnetization 
values $(\mathrm{Ms}=82.8 \mathrm{emu} / \mathrm{g}$ ) of the as-prepared functionalized MNPs measured at ambient temperature, as well as their superparamagnetic behavior, make them attractive candidates for a variety of applications, including heat transfer, magnetic separation, and magnetic hyperthermia. Due to the presence of the free amine group on the MNP's surface, they are excellent candidates for linking various applicatory functions. Exploiting this fact, MNP-AIm_1 and MNP-AIm_2 were used as a platform for PBAAA covalent linkage, increasing their stability, improving their colloidal stability. The ability of the amino groups on the MNP's surface from MNP-AIm_1 to link different molecules enhances their potential candidacy for immobilization of various moieties with biological or catalytic importance.

Author Contributions: Conceptualization, A.N.; Data curation, S.M. and R.T.; Formal analysis, A.N.; Funding acquisition, A.N. and R.T.; Investigation, A.N., I.-V.G. and R.T.; Methodology, A.N.; Project administration, A.N. and R.T.; Resources, A.N. and R.T.; Supervision, A.N. and R.T.; Writing-original draft, A.N., I.-V.G., S.M. and R.T.; Writing-review \& editing, A.N. and R.T. All authors have read and agreed to the published version of the manuscript.

Funding: This work was supported by a grant of the Ministry of Research, Innovation and Digitization, CNCS/CCCDI - UEFISCDI, project number PN-III-P4-ID-PCE-2020-1595, within PNCDI III.

Data Availability Statement: No new data were created or analyzed in this study. Data sharing is not applicable to this article.

Acknowledgments: The authors want to acknowledge Cristian Leostean, Monica Dan and Septimiu Tripon from the National Institute for Research and Development of Isotopic and Molecular Technologies Cluj-Napoca for conducting VSM measurements, TGA, and TEM analysis, respectively.

Conflicts of Interest: Iolanda-Veronica Ganea is an employee of MDPI; however, she did not work for the journal Nanomaterials at the time of submission and publication.

\section{References}

1. Vibhute, P.S.; Mhaldar, P.M.; Shejwal, R.V.; Pore, M.D. Magnetic nanoparticles-supported palladium catalyzed Suzuki-Miyaura cross coupling. Tetrahedron Lett. 2020, 61, 151594. [CrossRef]

2. Yacob, Z.; Nan, A.; Liebscher, J. Proline-functionalized magnetic core-shell nanoparticles as efficient and recyclable organocatalysts for Aldol reactions. Adv. Synth. Catal. 2012, 354, 3259-3264. [CrossRef]

3. Sobhani, S.; Falatooni, Z.M.; Asadi, S.; Honarmand, M. Palladium-Schiff Base Complex Immobilized Covalently on Magnetic Nanoparticles as an Efficient and Recyclable Catalyst for Heck and Suzuki Cross-Coupling Reactions. Catal. Lett. 2015, 146, 255-268. [CrossRef]

4. Ganea, I.-V.; Nan, A.; Baciu, C.; Turcu, R. Effective removal of crystal violet dye using neoteric magnetic nanostructures based on functionalized poly(benzofuran-co-arylacetic acid): Investigation of the adsorption behaviour and reusability. Nanomaterials 2021, 11, 679. [CrossRef]

5. Ekinci, S.; Ilter, Z.; Ercan, S.; Çınar, E.; Çakmak, R. Magnetite nanoparticles grafted with murexide-terminated polyamidoamine dendrimers for removal of lead (II) from aqueous solution: Synthesis, characterization, adsorption and antimicrobial activity studies. Heliyon 2021, 7, e0660. [CrossRef]

6. Wu, A.; Zhao, X.; Wang, J.; Tang, Z.; Zhao, T.; Niu, L.; Yu, W.; Yang, C.; Fang, M.; Lv, H.; et al. Application of solid-phase extraction based on magnetic nanoparticle adsorbents for the analysis of selected persistent organic pollutants in environmental water: A review of recent advances. Crit. Rev. Environ. Sci. Technol. 2020, 51, 44-112. [CrossRef]

7. Ng, S.M.; Koneswaran, M.; Narayanaswamy, R. A review on fluorescent inorganic nanoparticles for optical sensing applications. RSC Adv. 2016, 6, 21624-21661. [CrossRef]

8. Gloag, L.; Mehdipour, M.; Chen, D.; Tilley, R.D.; Gooding, J.J. Advances in the Application of Magnetic Nanoparticles for Sensing. Adv. Mater. 2019, 31, e1904385. [CrossRef]

9. Jing, D.; Sun, L.; Jin, J.; Thangamuthu, M.; Tang, J. Magneto-optical transmission in magnetic nanoparticle suspensions for different optical applications: A review. J. Phys. D Appl. Phys. 2020, 54, 013001. [CrossRef]

10. Patitsa, M.; Karathanou, K.; Kanaki, Z.; Tzioga, L.; Pippa, N.; Demetzos, C.; Verganelakis, D.A.; Cournia, Z.; Klinakis, A. Magnetic nanoparticles coated with polyarabic acid demonstrate enhanced drug delivery and imaging properties for cancer theranostic applications. Sci. Rep. 2017, 7, 775. [CrossRef] [PubMed]

11. Vangijzegem, T.; Stanicki, D.; Laurent, S. Magnetic iron oxide nanoparticles for drug delivery: Applications and characteristics. Expert Opin. Drug Deliv. 2019, 16, 69-78. [CrossRef]

12. Landarani-Isfahani, A.; Moghadam, M.; Mohammadi, S.; Royvaran, M.; Moshtael-Arani, N.; Rezaei, S.; Tangestaninejad, S.; Mirkhani, V.; Mohammadpoor-Baltork, I. Elegant pH-responsive nanovehicle for drug delivery based on triazine dendrimer modified magnetic nanoparticles. Langmuir 2017, 33, 8503-8515. [CrossRef] [PubMed] 
13. Naumenko, V.; Nikitin, A.; Garanina, A.; Melnikov, P.; Vodopyanov, S.; Kapitanova, K.; Potashnikova, D.; Vishnevskiy, D.; Alieva, I.; Ilyasov, A.; et al. Neutrophil-mediated transport is crucial for delivery of short-circulating magnetic nanoparticles to tumors. Acta Biomater. 2020, 104, 176-187. [CrossRef]

14. Nan, A.; Turcu, R. Poly(1-vinylimidazole) grafted on magnetic nanoparticles-Attainment of novel nanostructures. Rev. Roum. Chim. 2020, 65, 611-616. [CrossRef]

15. Kahani, S.A.; Yagini, Z. A Comparison between Chemical Synthesis Magnetite Nanoparticles and Biosynthesis Magnetite. Bioinorg. Chem. Appl. 2014, 2014, 384984. [CrossRef] [PubMed]

16. Cruz-Vargas, J.; Belmont-Bernal, F.; Vera-De la Garza, C.G.; Mazariego, J.L.P.; González, R.W.G.; Henao-Holguin, L.V.; RojasMontoyad, I.D.; Guadarrama, P. Accelerated one-pot synthesis of coated magnetic nanoparticles from iron(II) as a single precursor. New J. Chem. 2018, 42, 13107-13113. [CrossRef]

17. Aslam, M.; Schultz, E.A.; Sun, T.; Meade, T.; Dravid, V.P. Synthesis of amine-stabilized aqueous colloidal iron oxide nanoparticles. Cryst. Growth Des. 2007, 7, 471-475. [CrossRef]

18. Kahani, S.; Jafari, M. A new method for preparation of magnetite from iron oxyhydroxide or iron oxide and ferrous salt in aqueous solution. J. Magn. Magn. Mater. 2009, 321, 1951-1954. [CrossRef]

19. Bajaj, B.; Malhotra, B.D.; Choi, S. Preparation and characterization of bio-functionalized iron oxide nanoparticles for biomedical application. Thin Solid Films 2010, 519, 1219-1223. [CrossRef]

20. Molina, P.; Tárraga, A.; Otón, F. Imidazole derivatives: A comprehensive survey of their recognition properties. Org. Biomol. Chem. 2012, 10, 1711. [CrossRef] [PubMed]

21. Sundberg, R.J.; Martin, R.B. Interactions of histidine and other imidazole derivatives with transition metal ions in chemical and biological systems. Chem. Rev. 1974, 74, 471-517. [CrossRef]

22. Nan, A.; Bunge, A.; Cîrcu, M.; Petran, A.; Hadade, N.D.; Filip, X. Poly(benzofuran-co-arylacetic acid)—A new type of highly functionalized polymers. Polym. Chem. 2017, 8, 3504-3514. [CrossRef]

23. Zhang, X.-Q.; Gong, S.-W.; Zhang, Y.; Yang, T.; Wang, C.-Y.; Gu, N. Prussian blue modified iron oxide magnetic nanoparticles and their high peroxidase-like activity. J. Mater. Chem. 2010, 20, 5110-5116. [CrossRef]

24. Wilson, D.; Langell, M. XPS analysis of oleylamine/oleic acid capped $\mathrm{Fe}_{3} \mathrm{O}_{4}$ nanoparticles as a function of temperature. Appl. Surf. Sci. 2014, 303, 6-13. [CrossRef]

25. Poulin, S.; França, R.; Moreau-Bélanger, L.; Sacher, E. Confirmation of X-ray Photoelectron Spectroscopy Peak Attributions of Nanoparticulate Iron Oxides, Using Symmetric Peak Component Line Shapes. J. Phys. Chem. C 2010, 114, 10711-10718. [CrossRef]

26. Lei, L.; Shan, J.; Hu, J.; Liu, X.; Zhao, J.; Tong, Z. Co-curing effect of imidazole grafting graphene oxide synthesized by one-pot method to reinforce epoxy nanocomposites. Compos. Sci. Technol. 2016, 128, 161-168. [CrossRef]

27. Meiorin, C.; Muraca, D.; Pirota, K.R.; Aranguren, M.I.; Mosiewicki, M.A. Nanocomposites with superparamagnetic behavior based on a vegetable oil and magnetite nanoparticles. Eur. Polym. J. 2014, 53, 90-99. [CrossRef]

28. Cendrowski, K.; Sikora, P.; Zielinska, B.; Horszczaruk, E.; Mijowska, E. Chemical and thermal stability of core-shelled magnetite nanoparticles and solid silica. Appl. Surf. Sci. 2017, 407, 391-397. [CrossRef] 